\title{
Identification of a Catalytically Highly Active Surface Phase for CO Oxidation over PtRh Nanoparticles under Operando Reaction Conditions
}

\author{
U. Hejral, ${ }^{1,2,3, \dagger}$ D. Franz, ${ }^{1,2}$ S. Volkov, ${ }^{1,2}$ S. Francoual, ${ }^{1}$ J. Strempfer, ${ }^{1}$ and A. Stierle ${ }^{1,2, *}$ \\ ${ }^{1}$ Deutsches Elektronen-Synchrotron DESY, 22603 Hamburg, Germany \\ ${ }^{2}$ Fachbereich Physik, Universität Hamburg, 20355 Hamburg, Germany \\ ${ }^{3}$ Synchrotron Radiation Research, Lund University, 22100 Lund, Sweden
}

(Received 13 November 2017; published 23 March 2018)

\begin{abstract}
Pt-Rh alloy nanoparticles on oxide supports are widely employed in heterogeneous catalysis with applications ranging from automotive exhaust control to energy conversion. To improve catalyst performance, an atomic-scale correlation of the nanoparticle surface structure with its catalytic activity under industrially relevant operando conditions is essential. Here, we present x-ray diffraction data sensitive to the nanoparticle surface structure combined with in situ mass spectrometry during near ambient pressure CO oxidation. We identify the formation of ultrathin surface oxides by detecting x-ray diffraction signals from particular nanoparticle facets and correlate their evolution with the sample's enhanced catalytic activity. Our approach opens the door for an in-depth characterization of well-defined, oxide-supported nanoparticle based catalysts under operando conditions with unprecedented atomic-scale resolution.
\end{abstract}

DOI: 10.1103/PhysRevLett.120.126101

Heterogeneous catalysts represent an essential class of materials involved in most of the chemical production processes as well as energy storage, conversion, and exhaust gas cleaning [1,2]. The importance of these processes for today's society triggers the need for an indepth, atomic-scale understanding of the underlying catalytic reactions [3]. This is hampered by the structural heterogeneity and dynamical complexity of real catalysts composed of monometallic and alloy nanoparticles on highly branched nanoscale oxide supports. The low metal concentration of typically a few weight percent and the nanoparticles' random orientation makes a structural and chemical investigation under realistic catalytic reaction conditions very demanding [4,5]. Especially, the atomic structure of nanoparticle surfaces is not accessible by most methods under realistic reaction conditions.

$4 d$ and $5 d$ transition metals, such as $\mathrm{Pt}, \mathrm{Rh}, \mathrm{Pd}$, and their alloys, are catalyst materials with applications ranging from energy conversion to automotive exhaust control $[1,6,7]$. Experiments on single crystal surfaces under applicationrelevant near-ambient pressure reaction conditions provided novel insight into the prototypical $\mathrm{CO}$ oxidation reaction mechanism at the atomic scale [8-12]. However, it is controversially discussed to date if extended ultrathin oxide layers or chemisorbed oxygen represent the catalytically most active phase for CO oxidation [12-19]. Besides, the information obtained from single crystal surfaces can not always be transferred to realistic nanoparticle containing catalysts since catalytic activity and selectivity are strongly affected by the interplay with the support and nanoscale properties [20,21]. These include the particle shape and size, undercoordinated atoms at corners and edges, substrate induced strain, or characteristic defects [22-24]. Recent operando transmission electron microscopy experiments provided first microscopic insight into nanoparticle surface structural changes under $\mathrm{CO}$ oxidation conditions but also evidenced the challenges of such experiments [25-27].

Here, we present $\mathrm{x}$-ray diffraction results from $\mathrm{Pt}_{0.33} \mathrm{Rh}_{0.67}$ alloy nanoparticles supported on $\mathrm{MgAl}_{2} \mathrm{O}_{4}(001)$, obtained during $\mathrm{CO}$ oxidation at near-ambient pressures and elevated temperatures. Our high resolution x-ray reciprocal space maps disclose a truncated octahedral particle shape composed of predominantly (111) and (100) type nanofacets under both, reducing and reaction conditions. We identify the formation of ultrathin surface oxides by detecting selective $\mathrm{x}$-ray diffraction signals from particular nanoparticle facets and find a direct correlation between the facetresolved $\mathrm{Rh}$ surface oxide formation and an enhanced $\mathrm{CO}$ oxidation activity monitored by online mass spectrometry (MS). Our experiments provide the first direct structural identification of a catalytically active phase on selected nanoparticle facets under steady state operando conditions.

The $\mathrm{MgAl}_{2} \mathrm{O}_{4}(001)$ substrate surface was prepared by subsequent thermal cracking $\left(p_{\mathrm{O} 2}=1 \times 10^{-7} \mathrm{mbar}, T=\right.$ $570 \mathrm{~K}$ ), sputtering ( $p_{\mathrm{Ar}}=1 \times 10^{-6} \mathrm{mbar}, U=1 \mathrm{keV}, I_{\text {ion }}=$ $3 \mu \mathrm{A}$ ), and annealing to $840 \mathrm{~K}$ at $p_{\mathrm{O} 2}=1 \times 10^{-7} \mathrm{mbar}$ [28]. Thereafter, nanoparticles were grown by the simultaneous physical vapor deposition of $\mathrm{Pt}$ and $\mathrm{Rh}$ at $770 \mathrm{~K}$. The sample was transported through air and mounted into an operando x-ray diffraction catalysis chamber [29,30]. Prior to the reaction experiment, the sample underwent hydrogen annealing ( $p_{\mathrm{H} 2}=2 \times 10^{-5} \mathrm{mbar}, T=620 \mathrm{~K}$ ) to remove residual oxides. For the experiments, pure $\mathrm{O}_{2}, 10 \% \mathrm{CO}$ in 
90\% Ar, and pure $\mathrm{Ar}$ as carrier gas were employed. The total pressure and the total gas flow were held constant at $50 \mathrm{mbar}$ and $50 \mathrm{ml}_{n} / \mathrm{min}$, respectively, by adjusting the Ar flow. Four conditions were probed: (i) $4 \mathrm{ml}_{n} / \mathrm{min} \mathrm{CO}$, no $\mathrm{O}_{2}$, (ii) $4 \mathrm{ml}_{n} / \operatorname{min~CO}, 1 \mathrm{ml}_{n} / \min \mathrm{O}_{2}$, (iii) $4 \mathrm{ml}_{n} / \min \mathrm{CO}$, $2 \mathrm{ml}_{n} / \operatorname{min~}_{2}$, and (iv) $4 \mathrm{ml}_{n} / \operatorname{min~CO}, 3 \mathrm{ml}_{n} / \operatorname{min~O}_{2}$; the sample temperature was kept constant at $650 \mathrm{~K}$. A carbonyl trap was used in the $\mathrm{CO}$ line to prevent contamination of the sample surface. For the x-ray experiment, the chamber was mounted on the heavy load diffractometer at beam line P09, PETRA III, DESY [31]. A photon energy of $11.2 \mathrm{keV}$ was used with the incident angle of the $\mathrm{x}$-ray beam fixed at the critical angle for total external reflection of Pt $\left(0.4^{\circ}\right)$. Data were collected by a $\mathrm{NaI}$ scintillation counter and the measured intensities were dead-time- and absorber-corrected.

The orientation and size of the $\mathrm{Pt}_{0.33} \mathrm{Rh}_{0.67}$ particles were characterized by line scans through particle Bragg peaks along high-symmetry directions. The particles were found to grow predominantly in a cube-on-cube epitaxy on the $\mathrm{MgAl}_{2} \mathrm{O}_{4}(001)$ substrate with a lattice mismatch of $5 \%$. Preferentially oriented nanoparticles with a high surface coverage lead to an enhancement of the nanoparticle surface signal, making these experiments feasible down to an average particle size of about $5 \mathrm{~nm}$. The analysis of the anisotropic particle Bragg peak widths revealed an average initial height $\bar{H}$ of $6.3 \mathrm{~nm}$ and diameter $\bar{D}$ of $8.7 \mathrm{~nm}$ [32-34]. The full width at half maximum (FWHM) of the Gaussian particle size distribution typically amounts to $70 \%$ of its center value $\bar{D}$ [35]. Figure 1(a) shows a high-resolution reciprocal space map measured during catalytic activity [condition (iv)] in the $(H, K=2-H, L)$-plane centered around the particle (111) Bragg reflection. The diffraction pattern contains clear crystal truncation rod (CTR) signals oriented perpendicular to the (001)- and (111)-type facets. Accordingly, a truncated octahedral particle shape as depicted in Fig. 1(b) was deduced for both reducing and CO oxidation activity conditions [30,32,34].

Figures 1(c) and 1(d) show close-ups on the clean particle (001) and (111) facets and their surface unit cells. In addition, the $\mathrm{Rh} c(2 \times 8)$ and the $\mathrm{Rh} p(9 \times 9)$ trilayer surface oxide unit cells are given. Both were reported to form under oxygen exposure on Rh single crystal (001) and (111) surfaces and corresponding $\mathrm{MgO}(001)$-supported $\mathrm{Rh}$ nanoparticle facets [32,36,37], as well as during $\mathrm{CO}$ oxidation on single crystal surfaces $[38,39]$. An evidence of their presence, or even evolution, on distinct nanoparticle facets under various reaction conditions is, however, lacking so far. To track the presence of transient surface oxides on particular facets during the probed operando conditions, reference scans were performed. Figures 1(c) and 1(d) illustrate the reciprocal space maps of the particle (001)- and (111)-type facets corresponding for the (001) facets to a real space lattice with $a_{001}=b_{001}=2.713 \AA$ and $\gamma=90^{\circ}$ [for the (111) facets: $a_{001}=b_{001}=2.713 \AA, \gamma=120^{\circ}$ ]. (a)

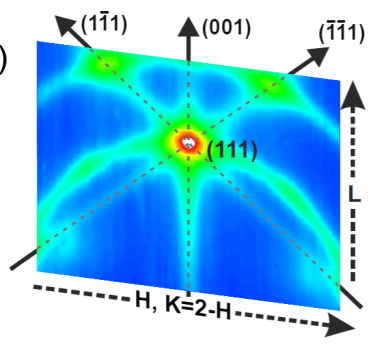

(b)
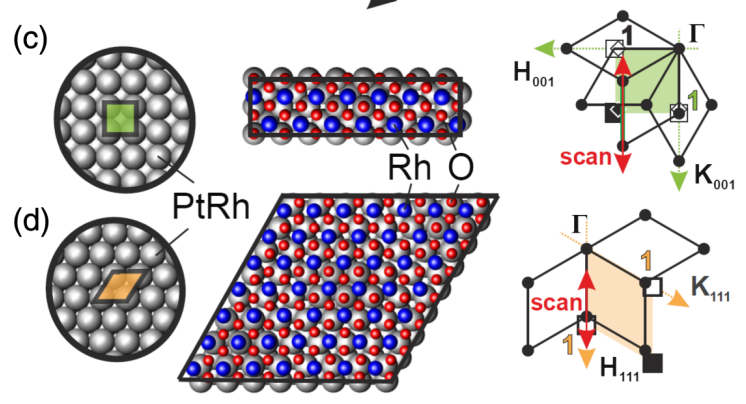

FIG. 1. (a) High-resolution reciprocal space map in the bulk fcc $(H, K=2-H, L)$ plane under reaction conditions, evidencing a truncated octahedral nanoparticle shape in (b) (see [30] for explanation of the additional diffraction features). (c) (001) type facet with surface unit cell indicated in green, the Rh $c(2 \times 8)$ surface oxide and reciprocal space in surface coordinates of the (001) facet. (d) (111) type facet with surface unit cell indicated in orange, the $\mathrm{Rh} p(9 \times 9)$ surface oxide and reciprocal space in surface coordinates. Filled and open squares: particle Bragg peaks and CTRs, respectively; red arrows: performed reference line scans.

In the following, the variation in gas composition and the resulting activity for $\mathrm{CO}_{2}$ production will be discussed. Figures 2(a)-2(c) show the MS signals for $\mathrm{CO}, \mathrm{O}_{2}$, and the reaction product $\mathrm{CO}_{2}$ during the stepwise increase of the



FIG. 2. MS data of (a) $\mathrm{CO}$, (b) $\mathrm{O}_{2}$, and (c) $\mathrm{CO}_{2}$ as a function of time and the set mass flows for conditions (i)-(iv). Left $y$ axis (black): MS signals measured by gas leaking into the chamber's UHV part. Right $y$ axis (red) (a) and (b): set mass flows. 
oxygen partial flow along with the set mass flows as a function of time. When introducing $\mathrm{O}_{2}$ to the reactor for the first time [transition from condition (i) to (ii)], an increase in the $\mathrm{CO}_{2}$ production was monitored, evidencing the sample's catalytic activity. It was accompanied by a decrease in the CO MS signal, in line with a partial mass transfer limitation of our setup under these conditions, giving rise to a partial depletion of both reactants near the sample surface [40]. In the next step [(ii) to (iii)], the $\mathrm{O}_{2}$ flow was doubled to stoichiometric conditions, leading to a pronounced $\mathrm{CO}_{2} \mathrm{MS}$ signal increase by a factor $\approx 2.5$, thereby reaching the sample's highest catalytic activity. At the same time, the decrease in the CO MS signal was more pronounced, as compared to the transition from step (i) to (ii), and the $\mathrm{O}_{2} \mathrm{MS}$ signal was not doubling, as would be expected when doubling the $\mathrm{O}_{2}$ flow, in line with an enhanced $\mathrm{CO}$ as well as mass transfer limited $\mathrm{O}_{2}$ consumption in this high activity regime. When switching to overstoichiometric oxygen conditions [(iii) to (iv)], the $\mathrm{CO}_{2}$ production decreased, a slight increase in the $\mathrm{CO}$ and a more pronounced increase in the $\mathrm{O}_{2}$ MS signal were visible, indicating a partial poisoning of the catalyst as less $\mathrm{CO}$ and $\mathrm{O}_{2}$ was consumed.

Next, we will discuss the structural changes of the nanoparticle facets as a function of the aforementioned gas compositions. The respective reference line scans for the Rh surface oxides on the (001) and (111) facets are plotted in Figs. 3(a), 3(b), and 3(c) for the probed conditions (i)-(iv). Under pure CO flow (i), as well as under oxygen understoichiometry (ii), no surface oxide signal was detected. For such reducing conditions, it is expected that $\mathrm{Pt}$ is slightly enriched at the surface, as compared to the bulk composition of the nanoparticles [41].

Under stoichiometric conditions (iii), two new reflections at $K \simeq 0.5$ and $K \simeq 1.5$ arose [Figs. 3(a) and 3(b)], both fingerprints of the ultrathin O-Rh-O trilayer $c(2 \times 8)$ surface oxide on the (001) top facet, see Fig. 3(d) [32,42]. Accordingly, under these more oxygen-rich conditions $\mathrm{Rh}$ segregation occurred, in line with a previous near ambient pressure XPS study [41]. The FWHM of the reflections yield a surface oxide domain size of $\approx 8 \mathrm{~nm}$. This is in good agreement with the nanoparticle (001) top facet width [see Fig. 3(e)] of the particles with a shape as determined from Bragg peak scans along high-symmetry directions performed under condition (iv) [30]. Note that the nanoparticles underwent $\mathrm{CO}$ oxidation induced sintering during the experiment [43]. This implies that the (001)-type facets are fully covered by the $\mathrm{Rh} c(2 \times 8)$ surface oxide under these conditions. Contrarily, no additional signal was observed in the (111) facet reference scan shown in Fig. 3(c), indicating the absence of the $\mathrm{Rh} p(9 \times 9)$ surface oxide on the (111) facets. An explanation can be found in the slightly higher heat of formation of the O-Rh-O trilayer surface oxide on the (001) facets [44].

When increasing the oxygen pressure to overstoichiometric conditions (iv), we made two surprising observations:
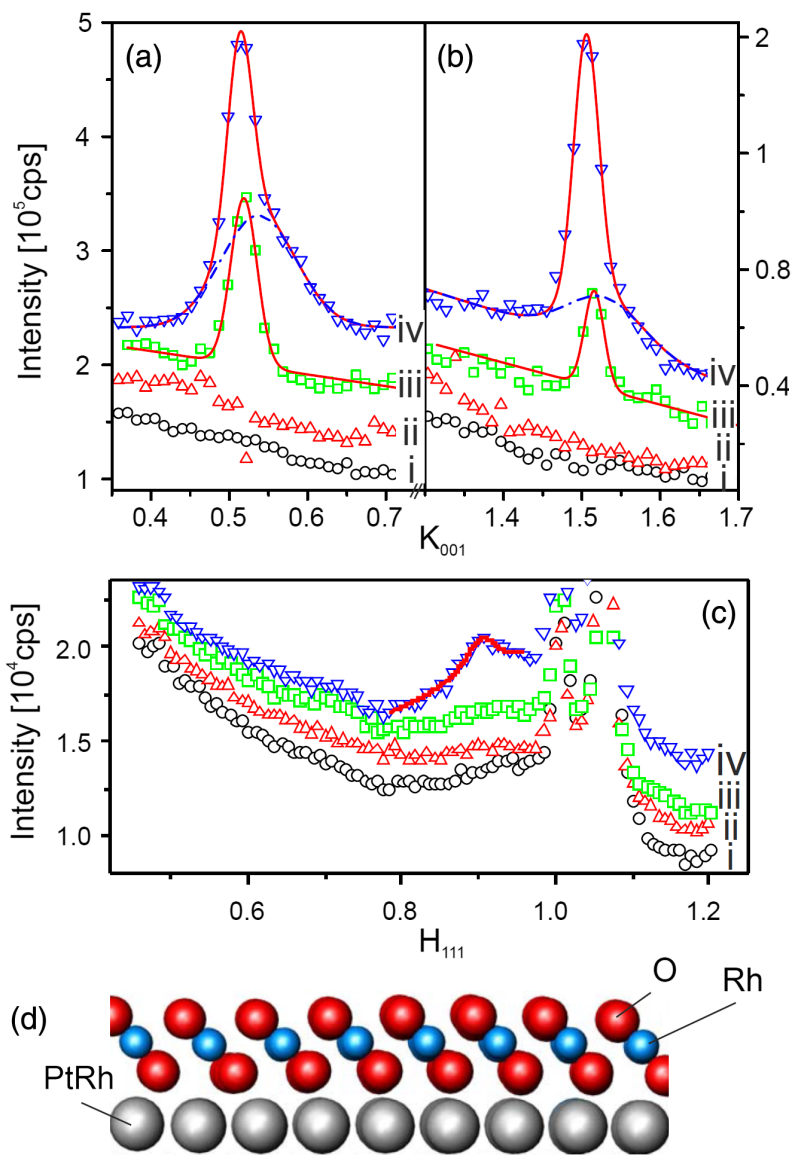

(e)

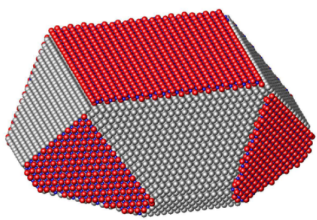

iii

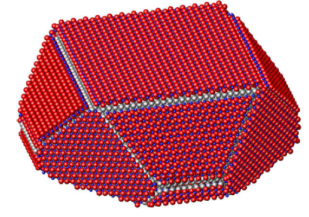

iv
FIG. 3. Reference scans for surface oxide formation: (a), (b) on the (001) top facets $\left(H_{001}=0.875\right)$, (c) on the (111) side facets $\left(K_{111}=0\right)$. Open circles: pure CO (i). Upward triangles: understoichiometric in $\mathrm{O}_{2}$ (ii). Squares: stoichiometric conditions (iii). Downward triangles: overstoichiometric in $\mathrm{O}_{2}$ (iv). Scans are rescaled for clarity. Solid (red) lines represent fits to the data (see text). (d) O-Rh-O trilayer surface oxide present on the (001) and (111) facets. (e) Average particle shape as determined from fitting the asymmetrically broadened Bragg peak line scans for condition iv: $\bar{D}=11.6 \mathrm{~nm}, \bar{H}=4.0 \mathrm{~nm}$ [30].

(1) a distinct peak was observed in the line scan in Fig 3(c) at $H=0.9$, in line with a $\mathrm{O}-\mathrm{Rh}-\mathrm{O}$ trilayer surface oxide on the $(111)$ facets $[32,36]$. The peak width corresponds to a domain size of about $4 \mathrm{~nm}$, reflecting the width of the $\mathrm{Rh}$ $p(9 \times 9)$ surface oxide domains on the (111) facets along the scan direction. (2) The overall shape of the reference scan for the (001) facets also changed [see Figs. 3(a) and 3(b)]: at $H \simeq 0.54$ an additional component arose, leading to the overall intensity increase of the $c(2 \times 8)$ surface oxide signal centered around $H \simeq 0.5$, at $H \simeq 1.5$ the signal increased, 
and at $H \simeq 1.53$ a small shoulder appeared. These three additional components correspond to the respective $d$-spacings of $2.64 \AA, 1.57 \AA$, and $1.50 \AA$, in line with the formation of $\mathrm{RhO}_{2}$ and $\mathrm{Rh}_{3} \mathrm{O}_{4}$ bulk oxide islands [30,35], coexisting with the surface oxides. For the fully oxide-covered PtRh nanoparticles, a slight decrease in $\mathrm{CO}_{2}$ production was observed, as can be deduced from Fig. 2(c).

To obtain insights into the dynamics of the formation and dissolution of the different oxide phases and their stability under varying conditions, we performed time-resolved operando gas-switching experiments: the diffracted intensities at $H_{001}=0.875, K_{001}=0.51(\mathrm{Rh} c(2 \times 8)$ surface oxide on the (001) facets and $\mathrm{RhO}_{2}$ bulk oxide) and at $H_{111}=0.89, K_{111}=0[p(9 \times 9)$ surface oxide on the $(111)$ facets] were monitored during an identical gas switching sequence (iv)-(iii)-(ii)-(i)-(ii)-(iii)-(iv) at a constant temperature of $650 \mathrm{~K}$. Figures 4(a) and 4(b) show the obtained diffraction signals of the (001) top and (111) side facets (green curves) plotted along with the set gas flows and the measured partial gas pressures. Starting at overstoichiometric conditions (iv), the monitored intensities indicate that, under these conditions, all surface and bulk oxides were present. In the transition to stoichiometric conditions (iii), no significant change was observed in the diffraction signals, pointing to a hysteresis in the stability of the surface and transient bulk oxides. Only when switching to understoichiometric conditions in $\mathrm{O}_{2}$ [(ii) and (i)], the surface oxides and transient bulk oxides were lifted instantaneously on both types of facets, and the $\mathrm{CO}_{2}$ production was observed to break down immediately.

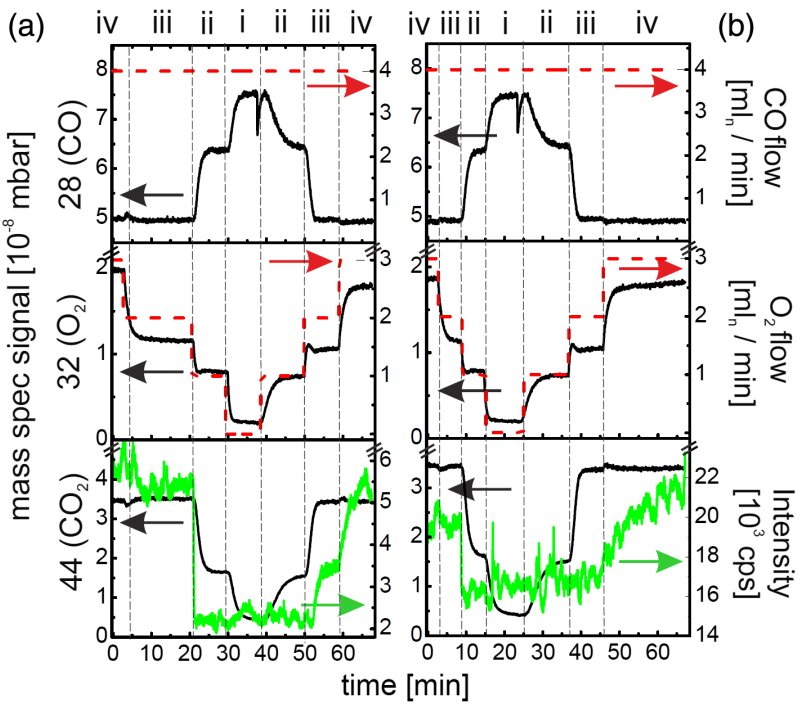

FIG. 4. Time-resolved MS data and x-ray diffraction signals at (a): $H_{001}=0.875, K_{001}=0.51$, at (b): $H_{111}=0.91, K_{111}=0$. Upper panels, left $y$ axis: measured CO MS signal (black solid lines), right $y$ axis: set $\mathrm{CO}$ flow (red dashed line); middle panels: left $y$ axis: measured $\mathrm{O}_{2}$ MS signal (black solid line), right $y$ axis: set $\mathrm{O}_{2}$ flow (red dashed line); bottom panels: left $y$ axis: measured $\mathrm{CO}_{2} \mathrm{MS}$ signal (black solid line), right $y$ axis (green solid line): diffracted intensity.
When going back from reducing conditions (ii) to stoichiometric conditions (iii), the enhanced increase in $\mathrm{CO}_{2}$ production correlated with an increase in the surface oxide signal of the (001) facets, whereas no increase of the (111) facet surface oxide diffraction signal was observed. The latter only increased slowly when switching from condition (iii) to conditions overstoichiometric in $\mathrm{O}_{2}$ (iv), suggesting that the most active state of the catalyst was already reached under stoichiometric conditions, with the surface oxide present on the (001) top facets only. At the same time, the diffraction signal in the (100) facet reference scan increased further in line with the above discussed formation of transient bulk oxide islands, without further increase in activity. During the switching cycle, reproducible mass transfer limitation effects were observed for the $\mathrm{CO}$ and $\mathrm{O}_{2}$ MS signals, as discussed above.

We interpret our observations in terms of adsorption kinetics and draw the following conclusions on the catalytically active phase for our experimental conditions: under understoichiometric conditions (ii) our data do not show the formation of any Rh oxide phases; it is, therefore, reasonable to assume that a Langmuir Hinshelwood (LH) type reaction mechanism is present, during which oxygen undergoes dissociative chemisorption and $\mathrm{CO}$ reacts with adsorbed oxygen to form $\mathrm{CO}_{2}$ [45]. The $\mathrm{O}_{2}$ pressure dependence of the $\mathrm{CO}_{2}$ formation rate is discussed in the literature to be first order $[45,46]$. When increasing the oxygen flow to stoichiometric conditions from 1 to $2 \mathrm{ml}_{n} / \mathrm{min}$ and the partial pressure from 1 to $2 \mathrm{mbar}$, we found that the $\mathrm{CO}_{2}$ production rate increased by a factor of 2.5. Strikingly, at the same time, the metallic surface area decreased by a factor $\simeq 2$, because all the (001) type facets were covered by an oxygen-terminated surface oxide layer, prohibiting $\mathrm{CO}$ adsorption and oxygen dissociation [38]. In sum, the activity increase is significantly higher than expected from the simple $\mathrm{LH}$ picture. A lowering of the $\mathrm{CO}$ oxidation barriers at the borders of the (001) facet surface oxide islands to the remaining metallic (111) PtRh facets may lead to the enhanced activity [38]. Our observations are in line with the kinetic study in Ref. [13], concluding that the most active phase for $\mathrm{CO}$ oxidation on $\mathrm{Rh}$ contains a significant amount of oxygen.

Interestingly, the $\mathrm{CO}_{2}$ production rate stayed nearly constant when increasing the oxygen flow to overstoichiometric conditions, resulting in further surface oxide growth on the (111) type facets. Likely, catalytically highly active sites are present at edges and corners of the nanoparticles, where the surface oxide layers on (001) type and (111) type facets can not grow together. The unsaturated metal and oxygen sites at the nanoparticle edges and corners serve in this picture as promoters for $\mathrm{CO}$ oxidation and the replenishing of the surface oxide. At the same time, a slight deactivation takes place by the growth of transient bulk oxides.

In conclusion, we resolved the facet atomic surface structure from $\mathrm{MgAl}_{2} \mathrm{O}_{4}(001)$ supported PtRh nanoparticles 
under industrially relevant $\mathrm{CO}$ oxidation conditions by surface $\mathrm{x}$-ray diffraction in combination with online mass spectrometry. By detecting x-ray diffraction signals from particular nanoparticle facets of defined model catalysts, we overcome the complexity of real catalysts' heterogeneity but gain, at the same time, essential insights into catalyst nanoscale properties. Thus, we can directly correlate the formation of an O-Rh-O trilayer surface oxide on the (001) facets with an increase in the $\mathrm{CO}_{2}$ production rate, which is higher than expected from a simple Langmuir Hinshelwood reaction mechanism. We assign this enhanced activity to the unique properties of the triple phase boundary between the gas phase, surface oxide, and free sites on the metallic surface at nanoparticle edges and corners, reflecting important aspects of real, heterogeneous catalysts. Future $a b$ initio kinetic Monte Carlo simulations may take these into account allowing a better comparison between operando experiments and theory [47]. Our approach can contribute to the knowledge-based future development of novel heterogeneous catalysts with enhanced activity, selectivity, and improved lifetime.

Fruitful discussions with Edvin Lundgren and Johan Gustafson are acknowledged. The authors thank D. Reuther for his technical support during the beam times at P09, PETRA III, Deutsches Elektronen-Synchrotron (DESY), a member of the Helmholtz Association (HGF). Financial support by the Bundesministerium für Bildung und Forschung (BMBF), Project No. 05K10PS1 (NanoXcat) is gratefully acknowledged.

*Corresponding author. andreas.stierle@desy.de ${ }^{\dagger}$ Corresponding author. uta.hejral@sljus.lu.se

[1] G. Ertl, H. Knözinger, F. Schüth, and J. Weitkamp, Handbook of Heterogeneous Catalysis, edited by G. Ertl, H. Knözinger, F. Schüth, and J. Weitkamp (Wiley-VCH, Weinheim, Germany, 2008).

[2] R. Schlögl, Angew. Chem., Int. Ed. Engl. 54, 3465 (2015).

[3] K. F. Kalz, R. Kraehnert, M. Dvoyashkin, R. Dittmeyer, R. Glaser, U. Krewer, K. Reuter, and J. D. Grunwaldt, ChemCatChem 9, 17 (2017).

[4] M. A. Newton, Chem. Soc. Rev. 37, 2644 (2008).

[5] A. Frenkel, Z. Kristallogr. 222, 605 (2007).

[6] J. Y. Park, Y. Zhang, M. Grass, T. Zhang, and G. A. Somorjai, Nano Lett. 8, 673 (2008).

[7] J. R. Renzas, W. Y. Huang, Y. W. Zhang, M. E. Grass, D. T. Hoang, S. Alayoglu, D. R. Butcher, F. Tao, Z. Liu, and G. A. Somorjai, Phys. Chem. Chem. Phys. 13, 2556 (2011).

[8] M. D. Ackermann, T. Pedersen, B. Hendriksen, O. Robach, S. Bobaru, I. Popa, C. Quiros, H. Kim, B. Hammer, S. Ferrer, and J. W. M. Frenken, Phys. Rev. Lett. 95, 255505 (2005).

[9] B. Hendriksen, S. Bobaru, and J. Frenken, Surf. Sci. 552, 229 (2004).
[10] J. Gustafson, M. Shipilin, C. Zhang, A. Stierle, U. Hejral, U. Ruett, O. Gutowski, P. A. Carlsson, M. Skoglundh, and E. Lundgren, Science 343, 758 (2014).

[11] H. Over, O. Balmes, and E. Lundgren, Surf. Sci. 603, 298 (2009).

[12] J. Gustafson, S. Blomberg, N. M. Martin, V. Fernandes, A. Borg, Z. Liu, R. Chang, and E. Lundgren, J. Phys. Condens. Matter 26, 055003 (2014).

[13] F. Gao, Y. Cai, K. K. Gath, Y. Wang, M. S. Chen, Q. L. Guo, and D. W. Goodman, J. Phys. Chem. C 113, 182 (2009).

[14] R. van Rijn, O. Balmes, R. Felici, J. Gustafson, D. Wermeille, R. Westerstrom, E. Lundgren, and J. W. M. Frenken, J. Phys. Chem. C 114, 6875 (2010).

[15] F. Gao, S. McClure, M. S. Chen, and D. W. Goodman, J. Phys. Chem. C 114, 22369 (2010).

[16] J. Gustafson, R. Westerström, O. Balmes, A. Resta, R. van Rijn, X. Torrelles, C. T. Herbshleb, J. W. M. Frenken, and E. Lundgren, J. Phys. Chem. C 114, 22372 (2010).

[17] D. R. Butcher, M. E. Grass, Z. H. Zeng, F. Aksoy, H. Bluhm, W. X. Li, B. S. Mun, G. A. Somorjai, and Z. Liu, J. Am. Chem. Soc. 133, 20319 (2011).

[18] M. S. Chen, Y. Cal, Z. Yan, K. K. Gath, S. Axnanda, and D. W. Goodman, Surf. Sci. 601, 5326 (2007).

[19] D. J. Miller, H. Öberg, S. Kaya, H. Sanchez Casalongue, D. Friebel, T. Anniyev, H. Ogasawara, H. Bluhm, L. G. M. Pettersson, and A. Nilsson, Phys. Rev. Lett. 107, 195502 (2011).

[20] B. R. Cuenya and F. Behafarid, Surf. Sci. Rep. 70, 135 (2015).

[21] S. Schauermann, N. Nilius, S. Shaikhutdinov, and H.-J. Freund, Acc. Chem. Res. 46, 1673 (2013).

[22] H. Mistry, F. Behafarid, E. Zhou, L. K. Ono, L. Zhang, and B. R. Cuenya, ACS Catal. 4, 109 (2014).

[23] C. Henry, Surf. Sci. Rep. 31, 231 (1998).

[24] I. Lee, R. Morales, M. A. Albiter, and F. Zaera, Proc. Natl. Acad. Sci. 105, 15241 (2008).

[25] S. B. Vendelbo, C. F. Elkjaer, H. Falsig, I. Puspitasari, P. Dona, L. Mele, B. Morana, B. J. Nelissen, R. van Rijn, J. F. Creemer, P. J. Kooyman, and S. Helveg, Nat. Mater. 13, 884 (2014).

[26] H. Yoshida, Y. Kuwauchi, J. R. Jinschek, K. Sun, S. Tanaka, M. Kohyama, S. Shimada, M. Haruta, and S. Takeda, Science 335, 317 (2012).

[27] F. F. Tao and P. A. Crozier, Chem. Rev. 116, 3487 (2016).

[28] A. Stierle, T. F. Keller, H. Noei, V. Vonk, and R. Röhlsberger, J. Large-scale Res. Facilities 2, A76 (2016).

[29] R. van Rijn, M. D. Ackermann, O. Balmes, T. Dufrane, A. Geluk, H. Gonzalez, H. Isern, E. de Kuyper, L. Petit, V. A. Sole, D. Wermeille, R. Felici, and J. W. M. Frenken, Rev. Sci. Instrum. 81, 014101 (2010).

[30] See Supplemental Material at http://link.aps.org/ supplemental/10.1103/PhysRevLett.120.126101 for a description of the experimental setup, quantitative particle shape determination, and identification of bulk oxide phases.

[31] J. Strempfer, S. Francoual, D. Reuther, D. K. Shukla, A. Skaugen, H. Schulte-Schrepping, T. Kracht, and H. Franz, J. Synchrotron Radiat. 20, 541 (2013). 
[32] P. Nolte, A. Stierle, N. Y. Jin-Phillipp, N. Kasper, T. U. Schulli, and H. Dosch, Science 321, 1654 (2008).

[33] N. Kasper, A. Stierle, P. Nolte, Y. Jin-Phillipp, T. Wagner, D. G. de Oteyza, and H. Dosch, Surf. Sci. 600, 2860 (2006).

[34] U. Hejral, A. Vlad, P. Nolte, and A. Stierle, J. Phys. Chem. C 117, 19955 (2013).

[35] P. Müller, U. Hejral, U. Rütt, and A. Stierle, Phys. Chem. Chem. Phys. 16, 13866 (2014).

[36] J. Gustafson, A. Mikkelsen, M. Borg, E. Lundgren, L. Kohler, G. Kresse, M. Schmid, P. Varga, J. Yuhara, X. Torrelles, C. Quiros, and J. N. Andersen, Phys. Rev. Lett. 92, 126102 (2004).

[37] J. Gustafson, A. Mikkelsen, M. Borg, J. N. Andersen, E. Lundgren, C. Klein, W. Hofer, M. Schmid, P. Varga, L. Kohler, G. Kresse, N. Kasper, A. Stierle, and H. Dosch, Phys. Rev. B 71, 115442 (2005).

[38] R. Westerström, J. G. Wang, M. D. Ackermann, J. Gustafson, A. Resta, A. Mikkelsen, J. N. Andersen, E. Lundgren, O. Balmes, X. Torrelles, J. W. M. Frenken, and B. Hammer, J. Phys. Condens. Matter 20, 184018 (2008).
[39] J. Gustafson, R. Westerström, O. Balmes, A. Resta, R. van Rijn, X. Torrelles, C. T. Herbschleb, J. W. M. Frenken, and E. Lundgren, J. Phys. Chem. C 114, 4580 (2010).

[40] H. Karadeniz, C. Karakaya, S. Tischer, and O. Deutschmann, Chem. Eng. Sci. 104, 899 (2013).

[41] F. Tao, M. E. Grass, Y. Zhang, D. R. Butcher, F. Aksoy, S. Aloni, V. Altoe, S. Alayoglu, J. R. Renzas, C.-K. Tsung, Z. Zhu, Z. Liu, M. Salmeron, and G. A. Somorjai, J. Am. Chem. Soc. 132, 8697 (2010).

[42] The surface oxide reflections are slightly shifted from their ideal positions, pointing to a distortion.

[43] U. Hejral, P. Muller, O. Balmes, D. Pontoni, and A. Stierle, Nat. Commun. 7, 10964 (2016).

[44] F. Mittendorfer, N. Seriani, O. Dubay, and G. Kresse, Phys. Rev. B 76, 233413 (2007).

[45] C. Peden, D. Goodman, D. Blair, P. Berlowitz, G. Fisher, and S. Oh, J. Phys. Chem. 92, 1563 (1988).

[46] S. Oh, G. Fisher, J. Carpenter, and D. Goodman, J. Catal. 100, 360 (1986).

[47] K. Reuter, Catal. Lett. 146, 541 (2016). 